\title{
Electro-optical Properties of Twisted Nematic Liquid Crystal Cell with Silver Nanowire Network Electrodes
}

\author{
Kyeong-Wook Jang*, Jeong-Min Han** and Jin-Geun Shon ${ }^{\dagger}$
}

\begin{abstract}
This paper introduces liquid crystal (LC) alignment and its electro-optical properties in the LC cells with silver nanowire (AgNW) networks. The AgNW network was used as an electrode of LC cell as a substitute for an indium-tin-oxide (ITO) film. LC alignment characteristics in the LC cell using AgNW networks, which have two different sheet resistances of $60 \Omega / \mathrm{m}^{2}$ and $80 \Omega / \mathrm{m}^{2}$, were observed. The LC alignment characteristics including pretilt angle, LC alignment state, and thermal stability are similar irrespective of sheet resistance of AgNW network. However, twisted-nematic (TN)-LC cell normally operated when using AgNW network with sheet resistance of $80 \Omega / \mathrm{m}^{2}$. Electrooptical properties of TN-LC cell exhibited competitive performance compared to those of TN-LC cell based on conventional ITO electrode, which allow new approaches to replace conventional ITO electrode in display technology.
\end{abstract}

Keywords: Electrode, Electro-optical properties, Liquid crystal alignment, Silver nanowire

\section{Introduction}

Generally, metals are well known as the most conductive materials on earth due to their high density of free electrons. However, metals are also highly reflective in the visible wavelength region and not transparent. Their reflexibilities can be overcome by reducing the dimensions (smaller than visible wavelengths) while maintaining good electrical conductivity $[1,2]$. Among the various metals, silver nanowires (AgNWs) have attracted considerable interest because of their exceptional electrical, physical and mechanical properties from academia to industry over the past few years [1-12]. In particular, transparent conducting film (TCO) based on AgNWs with low sheet resistance and high transmittance has been exploited for wide range of optoelectronic devices and components such as solar cells [3-5], touch panels [6, 7], organic light-emitting diodes (OLEDs) [8-10], and liquid crystal displays (LCDs) [11].

Transparent electrodes composed of AgNW networks can be easily achieved by simple and scalable solution process using $\mathrm{AgNW}$ dispersions. These $\mathrm{AgNW}$ networks can substitute for the indium-tin-oxide (ITO) electrode in LCD applications. Some research groups reported substitutability of AgNW network instead of ITO film through the recent work that AgNW networks can be applied to organic solar cells. These results also indicates that the AgNW networks can form chemically stable interfaces with polymer layers $[12,13]$. Therefore, it is

$\dagger$ Corresponding Author: Department of Electrical Engineering, Gachon University, Korea. (shon@gachon.ac.kr)

* Department of Electrical Engineering, Gachon University, Korea (jkw7397@naver.com)

** Department of Electronic, Seoil University, Korea. (hanjm@seoil.ac.kr)

Received: December 3, 2015; Accepted: March 6, 2016 expected that the use of AgNW networks, located under a polyimide (PI) layers for liquid crystal (LC) alignment, can replace the ITO electrode in LCDs.

In this study, we used AgNW networks as LCD electrodes, in place of the ITO films. The electrical, optical, and morphological properties of the AgNW networks were investigated. In addition, the LC alignment characteristics including LC alignment states, pretilt angles, and thermal stabilities were observed by applying the AgNW network in LC cells. Finally, electro-optical (EO) characteristics of twisted-nematic (TN) LC cells were measured to confirm the potential of the AgNW networks to be applied as the electrodes of LCD applications.

\section{Experiments}

Prior to deposition of AgNW networks, the glass substrates (Samsung Corning 1737) were cleaned supersonically in acetone, isopropyl alcohol, and deionized water sequentially for $10 \mathrm{~min}$; they were, then dried using $\mathrm{N} 2$ gas. Then AgNW networks were deposited using a bar coating with Meyer bars on the glass substrates instead of ITO film as an electrode in LCD applications. The AgNWs were annealed at $140{ }^{\circ} \mathrm{C}$ for $5 \mathrm{~min}$. The used AgNWs were received from Cambrios ClearOhm as suspensions in isopropyl alcohol. Sheet resistances of the AgNW networks were measured by using a four-point probe system and the $\mathrm{AgNW}$ network surfaces were observed by field-emission scanning electron microscope (FE-SEM) (S-4200, Hitachi). Homogeneous PI (SE-7492, Nissan chemical) was evenly coated on the AgNW networks to form LC alignment layers using a spin-coating method. The conditions of prebaking and imidizing for $\mathrm{LC}$ alignment were $80^{\circ} \mathrm{C}$ for 
$10 \mathrm{~min}$ and $230{ }^{\circ} \mathrm{C}$ for $1 \mathrm{~h}$. The thickness of the coated PI layer was approximately $50 \mathrm{~nm}$. We implemented the unidirectional rubbing method using velvet on the PI layers to promote homogeneous alignment. The rubbing strength (RS) was conventionally optimized and set at $300 \mathrm{~mm}$. The definition of the RS has been given in previous papers [14]. These substrates were fabricated in an antiparallel configuration and twisted-nematic $(\mathrm{TN})$ cells with cell gaps of of $60 \mu \mathrm{m}$ and $5 \mu \mathrm{m}$, respectively. Empty cells were then injected with polytive LCs (MJ001929, Merck), which had a reflective index $(\Delta \mathrm{n})$ of 0.077 , an isotropic transition temperature of $72{ }^{\circ} \mathrm{C}$ and a dielectric anisotropy $\Delta \varepsilon\left(\varepsilon_{\|}-\right.$ $\left.\varepsilon_{\perp}\right)$ of 8.2. The LC alignment characteristics and thermal stabilities were observed under a photomicroscope (BXP 51 , Olympus), and the pretilt angle was measured by the crystal rotation method (TBA 107 device, Autronic) [15]. The transmittances of the LC cells fabricated by AgNW networks were measured using a UV-VIS-NIR spectrometer (V-650, JASCO Co.) The EO characteristics of the TN cell were obtained by a LCD evaluation system (LCD 700, Otsuka Electronics).

\section{Result and Discussion}

Fig. 1 shows an FE-SEM image of the AgNW networks coated on the glass substrates by bar-coating. We compared the AgNW networks with two different sheet resistance of $60 \Omega / \mathrm{m}^{2}$ and $80 \Omega / \mathrm{m}^{2}$. The sheet resistance of the AgNW


Fig. 1. SEM images of AgNW networks on the glass substrates; (a) $60 \Omega / \mathrm{m}^{2}$ and (b) $80 \Omega / \mathrm{m}^{2}$ network can be controlled according to size of Mayer rod bar. As shown in Fig. 1(a), the AgNW networks are partially achieved. The aggregation of AgNWs was found, however sheet resistance of $60 \Omega / \mathrm{m}^{2}$ was measured evenly across the board. Whereas AgNW networks with a sheet resistance of $80 \Omega / \mathrm{m}^{2}$ showed an even distribution.

We measured the UV-Vis transmittance of the AgNW networks to evaluate its suitability for LCD electrode. Over the wavelength range of $380-780 \mathrm{~nm}$, the average optical transmittances of the AgNW networks with sheet resistances of $60 \Omega / \mathrm{m}^{2}$ and $80 \Omega / \mathrm{m}^{2}$ were $94.5 \%$ and $96.7 \%$, respectively. At the AgNW network with sheet resistance of $60 \Omega / \mathrm{m}^{2}$, the aggregation of AgNW bundles is biting into the transmittance of AgNW network. However, both trans-mittances of AgNW networks are comparable to that of commercial available ITO electrode (92.9\%). Therefore, the use of AgNW networks should result in no loss of

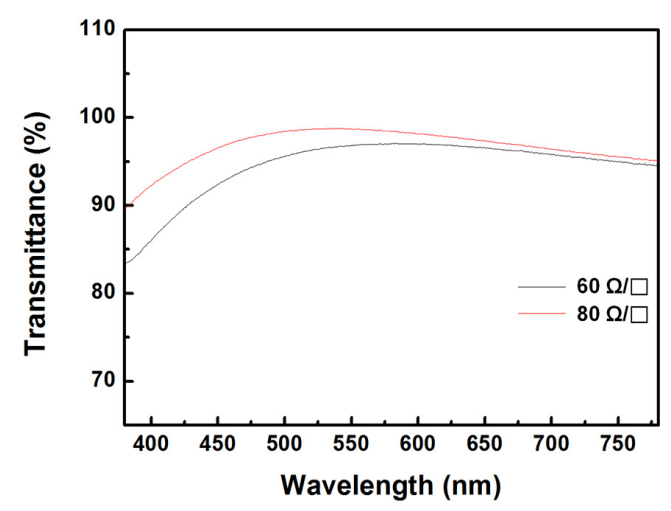

Fig. 2. UV-V is transmittance spectra of the AgNW networks $60 \Omega / \mathrm{m}^{2}$ and $80 \Omega / \mathrm{m}^{2}$

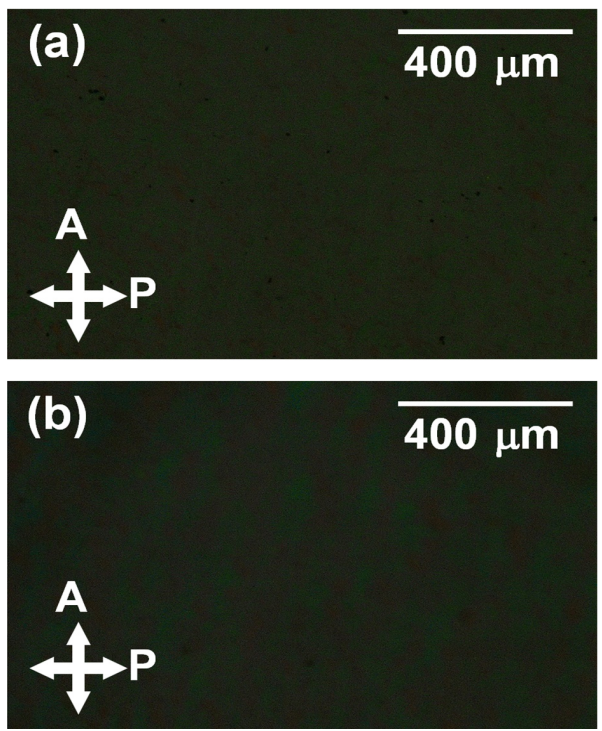

Fig. 3. Photomicrographs of LC cells based on AgNW networks with sheet resistance of (a) $60 \Omega / \mathrm{m}^{2}$ and (b) $80 \Omega / m^{2}$ under the crossed Nichols (A: analyzer, P: polarizer) 


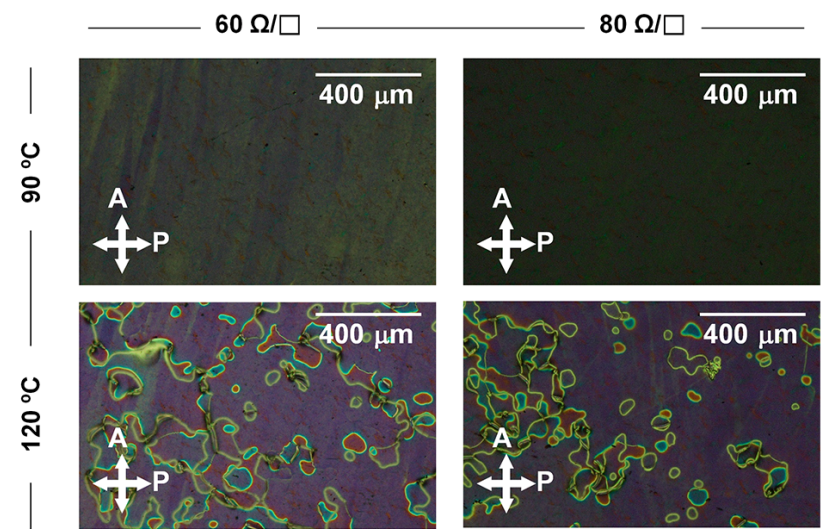

Fig. 4. Photomicrographs of LC cells using AgNW networks with sheet resistance of $60 \Omega / \mathrm{m}^{2}$ and 80 $\Omega / \mathrm{m}^{2}$ annealed at 90 and $120{ }^{\circ} \mathrm{C}$, respectively to examine thermal stability (A: analyzer, P: polarizer)

\section{transparency in LCD applications}

We observed electro-optical properties of LC cells to explore the feasibility of AgNW networks in LCD applications. The LC alignment statements for $\mathrm{LC}$ cells are important issues for high image quality in an LCD applications. Fig. 3 shows photomicrographs of LC cells with PI/AgNW networks. As shown in Fig. 3, uniform LC characteristics were achieved on both rubbed $\mathrm{PI} / \mathrm{AgNW}$ networks layers with sheet resistances of $60 \Omega / \mathrm{m}^{2}$ and 80 $\Omega / \mathrm{m}^{2}$ without any defects in the alignment. Good LC alignment was observed in both LC cells, indicating that uniform LC alignment was attributed to PI layers, not AgNW networks. In addition, we measured the pretilt angles of the LC cells using the crystal rotation method. The calculated pretilt angles of the LC molecules on the rubbed $\mathrm{PI} / \mathrm{AgNW}$ networks with sheet resistance of 60 $\Omega / m^{2}$ and $80 \Omega / m^{2}$ were $7.9^{\circ}$ and $7.8^{\circ}$, respectively.

Fig. 4 shows the photomicrographs of the LC cells using AgNW networks that were taken to examine the relationship between the ability of the LC molecules to maintain their orientation with respect to annealing temperature. Before the annealing process, LC cells were clear without any defects such as disclination or debris spots as shown in Fig. 3. The LC cells were annealed from 0 to $120^{\circ} \mathrm{C}$ for $10 \mathrm{~min}$ to examine the thermal stability of the LC cells. The LC molecules maintained uniformly stable alignment under the annealing temperature of $120^{\circ} \mathrm{C}$. However, LC orientations of the both LC cells were destroyed at an annealing temperature of $120{ }^{\circ} \mathrm{C}$. This result also indicates that the thermal budgets of LC cells sufficient to endure the thermal stress generated during the LCD operation and are not affected by AgNW networks.

EO characteristics were investigated to examine the potential of the AgNW network as an electrode for LCD application. Fig. 5(a) shows the voltage-transmittance curve of TN LC cells based on AgNW network with sheet resistance of $80 \Omega / \mathrm{m}^{2}$. The TN LC cells based on AgNW network with sheet resistance of $60 \Omega / \mathrm{m}^{2}$ operated
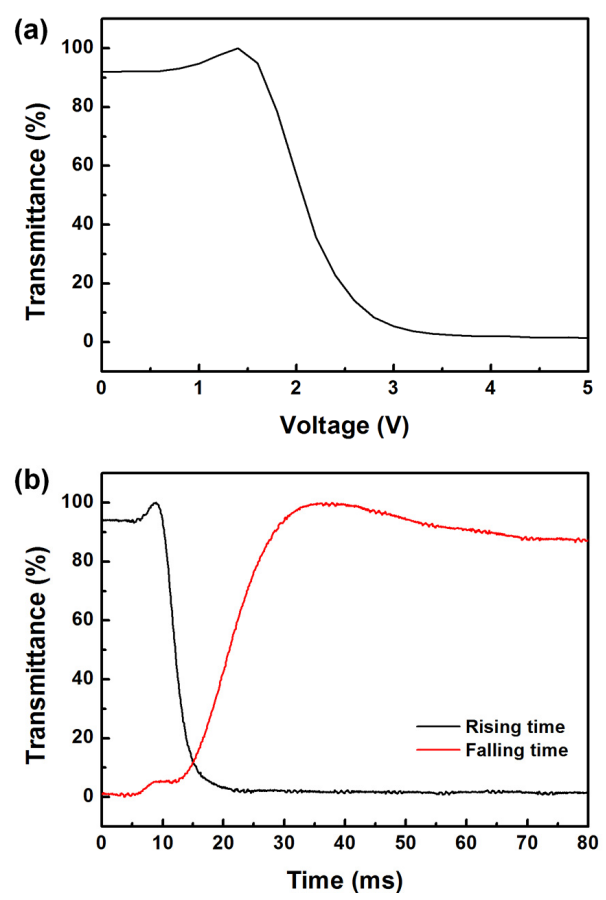

Fig. 5. (a) Voltage-transmittance and (b) response time characteristics of the TN LC cell fabricated with the $80 \Omega / \mathrm{m}^{2}$ AgNW networks

abnormally due to uneven distribution of AgNW bundles. The threshold voltage at a transmittance of $90 \%$ for TN LC cell with AgNW network was $1.66 \mathrm{~V}$, which value is similar to that of TN LC cell based on an ITO electrode. Fig. 5(b) shows the response time of TN LC cell with AgNW network. The response time of TN LC cell based on AgNW network with sheet resistance of $80 \Omega / \mathrm{m}^{2}$ was measured at $18.9 \mathrm{~ms}$ (rise time: $5.1 \mathrm{~ms}$, fall time: $13.8 \mathrm{~ms}$ ). This value is also the same level compared to conventional TN LC cell with ITO electrodes. As a result, the competitive advantages of LC cell fabricated with the AgNW networks suggest that AgNW network is appropriate as a substitute of conventional ITO electrode.

\section{Conclusion}

In conclusion, we measured LC alignment properties on the rubbed $\mathrm{PI} / \mathrm{AgNW}$ network layer, including pretilt angles, photomicrographs, and EO properties to observe the role of the AgNW network as an LCD electrode. The pretilt angles of LC molecules on the rubbed PI/AgNW network layers were $8^{\circ}$ irrespective of sheet resistance of AgNW network, which are applicable to TN LC cells. Uniform LC alignments were achieved in both LC cells and their thermal stabilities were similar to each other. Using a practical TN cell, competitive voltage-transmittance and response time characteristics were observed at AgNW network with sheet resistance of $80 \Omega / \mathrm{m}^{2}$ compared to TN LC cell with conventional ITO electrodes. These results 
demonstrate the high application potential of AgNW network as transparent electrodes of LCD application substituting conventional ITO electrodes.

\section{References}

[1] C.-H. Chung, T.-B. Song, B. Bob, R. Zhu, and Y. Yang, "Solution-processed flexible transparent conductors composed of silver nanowire networks embedded in indium tin oxide nanoparticle matrices" in Nano Res. 5, 805 (2012).

[2] S. M. Bergin, Y.-H. Chen, A. R. Rathmell, P. Charbonneau, Z.-Y. Li, and B. J. Wiley, "The effect of nanowire length and diameter on the properties of transparent, conducting nanowire films", Nanoscale 4, 1996 (2012).

[3] L. Hu, H. S. Kim, J.-Y. Lee, P. Peumans, and Y. Cui, "Scalable Coating and Properties of Transparent, Flexible, Silver Nanowire Electrode", ACS Nano 4, 2955 (2010).

[4] D.-S. Leem, A. Edwards, M. Faist, J. Nelson, D. D. C. Bradley, and J. C. de Mello, "Efficient Organic Solar Cells with Solution-Processed Silver Nanowire Electrodes", Adv. Mater. 23, 4371 (2011).

[5] N. E. Hjerrild, D. C. J. Neo, A. Kasdi, H. E. Assender, J. H. Warner, and A. A. R. Watt, "Transfer Printed Silver Nanowire Transparent Conductors for PbSZnO Heterojunction Quantum Dot Solar Cells", ACS Appl. Mater. Interfaces 7, 6417 (2015).

[6] J. Lee, P. Lee, H. B. Lee, S. Hong, I. Lee, J. Yeo, S. S. Lee, T.-S. Kim, D. Lee, and S. H. Ko, "RoomTemperature Nanosoldering of a Very Long Metal Nanowire Network by Conducting-Polymer-Assisted Joining for a Flexible Touch-Panel Application", Adv. Func. Mater. 23, 4171 (2013).

[7] Y. Kim, C.-H. Song, M.-G. Kwak, B.-K. Ju, and J.-W. Kim, "Flexible touch sensor with finely patterned Ag nanowires buried at the surface of a colorless polyimide film", RSC Adv. 5, 42500 (2015).

[8] X.-Y. Zeng, Q.-K. Zhang, R.-M. Yu, and C.-Z. Lu, "A New Transparent Conductor: Silver Nanowire Film Buried at the Surface of a Transparent Polymer", Adv. Mater. 22, 4484 (2010).

[9] W. Gaynor, S. Hofmann, M. G. Christoforo, C. Sachse, S. Mehra, A. Salleo, M. D. McGehee, M. C. Gather, B. Lüssem, L. Müller-Meskamp, P. Peumans, and K. Leo, "Color in the Corners: ITO-Free White OLEDs with Angular Color Stability", Adv. Mater. 25, 4006 (2013).

[10] Y.-H. Duan, Y. Duan, X. Wang, D. Yang, Y.-Q. Yang, P. Chen, F.-B. Sun, K.-W. Xue, and Y. Zhao, "Highly flexible peeled-off silver nanowire transparent anode using in organic light-emitting devices", Appl. Surf. Sci. 351, 445 (2015).

[11] L. Hu, H. Wu, and Y. Cui, "Metal nanogrids, nano- wires, and nanofibers for transparent electrodes", MRS Bulletin 36, 760 (2011).

[12] J.-Y. Lee, S. T. Connor, Y. Cui, and P. Peumans, "Semitransparent Organic Photovoltaic Cells with Laminated Top Electrode", Nano Lett. 10, 1276 (2010).

[13] F. Guo, P. Kubis, T. Stubhan, N. Li, D. Baran, T. Przybilla, E. Spiecker, K. Forberich, and C. J. Brabec, "Fully Solution-Processing Route toward Highly Transparent Polymer Solar Cells", ACS Appl. Mater. Interfaces 6, 18251 (2014).

[14] D.-S. Seo, S. Kobayashi, M. Nishikawa, "Study of the pretilt angle for $5 \mathrm{CB}$ on rubbed polyimide films containing trifluoromethyl moiety and analysis of the surface atomic concentration of $\mathrm{F} / \mathrm{C}(\%)$ with an electron spectroscope for chemical analysis", Appl. Phys. Lett. 61, 2392 (1992).

[15] T. J. Scheffer and J. Nehring, "Accurate determination of liquid-crystal tilt bias angles", J. Appl. Phys. 48, 1783 (1977).

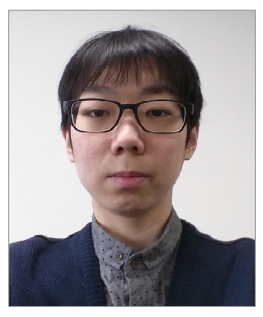

Kyeong-Wook Jang He received B.S. degrees in Department of Electrical Engineering, Gachon University, Gyeoggi-Do, Korea in 2015. He is currently a student of the Graduate School of Gachon University. His research interests are Material Science and Power conversion.

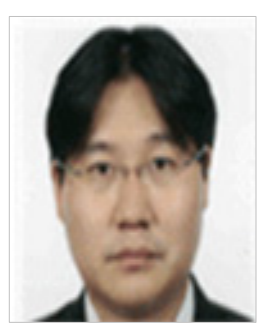

Jeong Min Han 2007, Ph. D, Electrical \& Electro Engineering, Graduate school, Yonsei University, 2009 Assistant Professor in Seoil University. Liquid Crystal Display, Material Science, Electro Display Device, Organic Material Science



Jin-Geun Shon He received his B.S., M.S. and Ph. D, degrees in the Department of Electrical Engineering from Soongsil University in 1990, 1992 and 1997. He is a Progessor at the school of Electrical Engineering, Gachon University, Korea. His research interests are the power conversion, control, LCD, and diagnosis of power utility 